
(1912)

\title{
La démonstration durkheimienne de Jean Price- Mars : faire du vodou haïtien une religion
}

The durkheimian demonstration of Jean Price-Mars: To turn Haitian voodoo into a religion

La prueba durkheimiana de Jean Price-Mars: hacer del vudú haitiano una religión

\section{Lewis Ampidu Clorméus}

\section{(2) OpenEdition Journals}

\section{Édition électronique}

URL : http://journals.openedition.org/assr/24140

DOI : $10.4000 /$ assr. 24140

ISSN : $1777-5825$

Éditeur

Éditions de l'EHESS

\section{Édition imprimée}

Date de publication : 30 septembre 2012

Pagination : 153-170

ISBN : 978-2-7132-2330-3

ISSN : 0335-5985

\section{Référence électronique}

Lewis Ampidu Clorméus, «La démonstration durkheimienne de Jean Price-Mars : faire du vodou haïtien une religion », Archives de sciences sociales des religions [En ligne], 159 | juillet-septembre 2012, mis en ligne le 26 novembre 2016, consulté le 10 décembre 2020. URL : http:// journals.openedition.org/assr/24140; DOI : https://doi.org/10.4000/assr.24140 


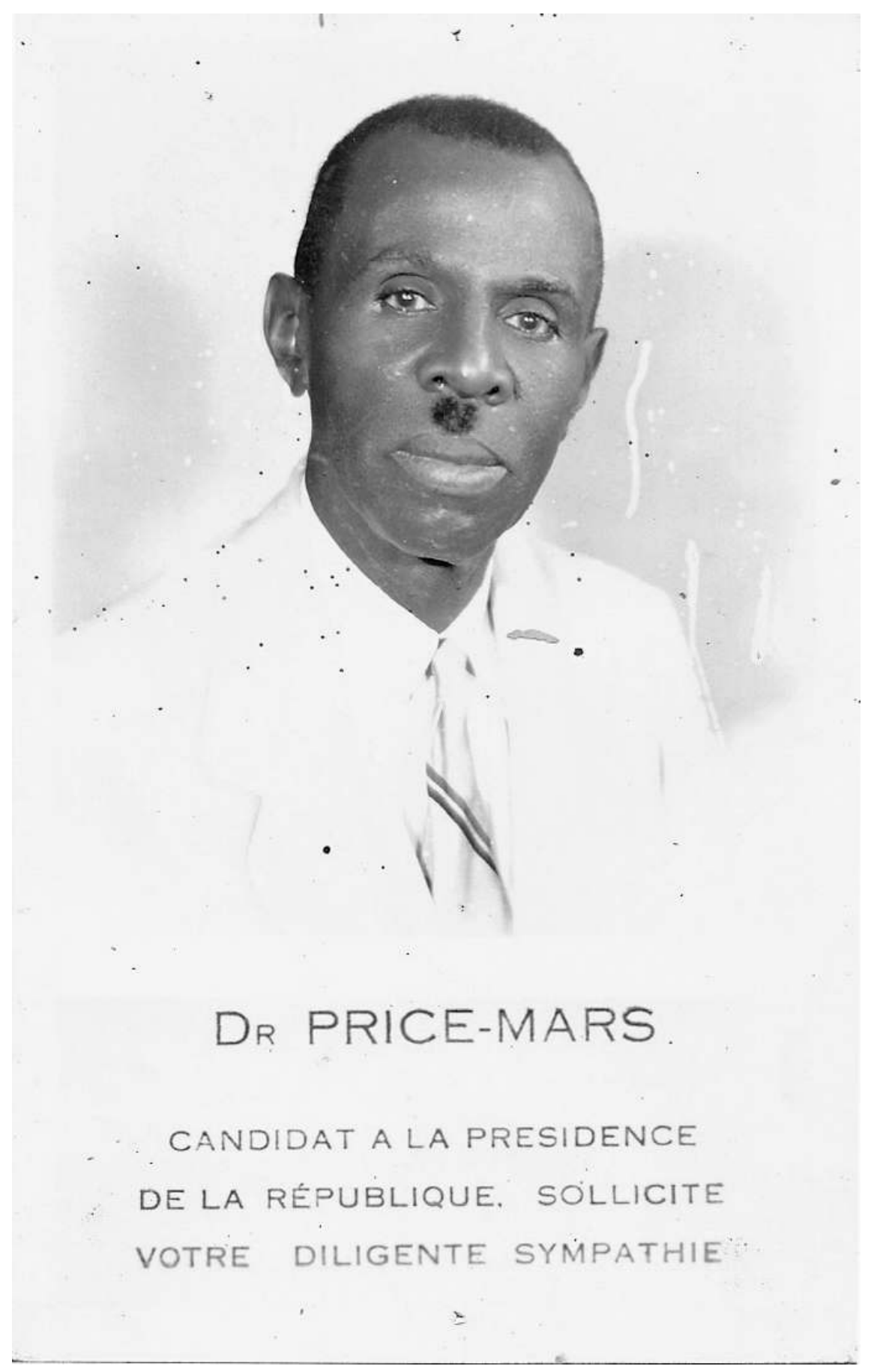

Jean Price-Mars (1876-1969) 


\section{Lewis Ampidu Clorméus}

\section{La démonstration durkheimienne de Jean Price-Mars : faire du vodou haïtien une religion}

Que vient faire la pensée d'Émile Durkheim dans la réhabilitation du vodou par Jean Price-Mars ? Cette question, autant qu'elle porte indirectement sur la constitution du discours indigéniste, renvoie à la réception tardive en Haïti des Formes élémentaires de la vie religieuse de Durkheim. Avant la publication d'Ainsi parla l'Oncle de Jean Price-Mars (1928), la pensée durkheimienne y est pratiquement méconnue. Le premier sociologue haïtien à évoquer le nom de Durkheim est probablement l'intellectuel positiviste Justin Dévot (1857-1921). Juriste, passionné par la sociologie comtienne, il a publié différents ouvrages de réflexion sur la société haïtienne. En 1905, le journal haïtien Le Nouvelliste a publié un extrait de ses Considérations sur la mentalité de la société haïtienne dans lequel il fait référence à Durkheim à propos des «états forts et définis de la conscience sociale » (Dévot, 1905 : 2). J. Dévot est peut-être même le premier intellectuel haïtien à enseigner la sociologie dans son pays ${ }^{1}$.

Qu'est-ce qui peut expliquer que le principal fondateur de la sociologie française soit si méconnu en Haïti avant 1928 ? Tout au cours du XIX ${ }^{\mathrm{e}}$ siècle, différents intellectuels haïtiens ont été membres de sociétés savantes parisiennes. Certains, comme Louis-Joseph Janvier ou Anténor Firmin, s'étaient illustrés par leur apport à la déconstruction du racisme scientifique en Europe. C'est peutêtre dans les luttes d'influence entre les cercles d'idées en France qu'il faut trouver une réponse à cette question. Durkheim s'impose dans la sociologie française à partir de la publication de La Division du travail social (Durkheim, 1893). Dès le départ, entre autres courants d'idées en vogue en France, la sociologie durkheimienne doit faire face à l'héritage leplaysien. Plusieurs Haïtiens, dès le tout début du Xx siècle, étaient membres de la revue La Science Sociale ${ }^{2}$ dirigée

1. Une note du journal Le Nomvelliste (1905c) rapporte qu'il devait inaugurer un cours de sociologie à l'École Nationale de Droit (Port-au-Prince) le samedi 28 octobre 1905. Voir aussi le compte rendu d'un étudiant en droit sur cet "événement " (Porsenna, 1905 : 1-2).

2. En 1907, parmi les membres de la Société Internationale de la Science Sociale, se distinguaient des adhérents domiciliés en Haïti. Parmi eux, citons le Frère Archange (Frères de l'Instruction Chrétienne), Mgr Conan (archevêque de Port-au-Prince), Luc Dominique (président du Cercle Catholique), Sœur Eugénie du Sacré-Cœur, Fleury Féquière (député de Portau-Prince), Eugène Roy, Me Pierre Hudicourt, Me Étienne Mathon (député), etc. 
par Edmond Demolins, un leplaysien devenu célèbre pour sa thèse sur la supériorité sociale anglo-saxonne (Demolins, 1897). Entre 1904 et 1910, une polémique même opposa les adeptes de Demolins aux partisans traditionnels de la francophilie en Haïti sur la nécessité et l'orientation d'une réforme sociale. Si ce débat n'a pas fait mention des travaux de Durkheim, la Grande Guerre (1914-1918) et l'Occupation américaine d'Haïti (1915-1934) n'ont guère facilité leur réception dans les milieux intellectuels de Port-au-Prince.

Aujourd'hui, il est impossible de retracer l'historique des discours littéraires et savants sur le vodou haïtien sans examiner Ainsi parla l'Oncle du docteur Jean Price-Mars. Dans ce livre, ce dernier propose de rompre avec des préjugés qui, depuis la période coloniale, ont entretenu une sinistre réputation à propos de croyances et pratiques religieuses afro-catholiques en Haïti. En s'inspirant de la définition durkheimienne de la religion, Price-Mars va provoquer un tournant dans l'histoire culturelle haïtienne ${ }^{3}$.

\section{2 ou l'année de la seconde campagne antisuperstitieuse en Haïti}

En 1912, l'année même de la parution du chef-d'œuvre de Durkheim, l'Église catholique lançait en Haïti sa seconde campagne antisuperstitieuse en Haïti. Il s'agit d'une offensive décidée par le clergé concordataire à dessein de combattre toute vision religieuse contraire à la doctrine catholique. Dans cette logique, c'est le vodou, assimilé à un ramassis de superstitions, qui est particulièrement visé parce qu'il incarne la persistance d'une primitivité religieuse et un obstacle à la civilisation.

Comment expliquer cette seconde campagne antisuperstitieuse ? Depuis le concordat du 28 mars 1860, le clergé catholique se charge officiellement de " civiliser» et de "moraliser" les masses haïtiennes par l'évangélisation et l'instruction. Malgré ses efforts, les résultats sont plutôt décevants. C'est d'ailleurs ce qu'atteste le contenu d'un livre d'un ancien chef de la Légation de France en Haïti (Aubin, 1910). Aussitôt publié, des intellectuels le considèrent comme un affront à l'image d'Haïti. Frédéric Marcelin reprocha à son auteur d'accorder trop de pages au «sempiternel vaudoux » qui n'est autre que "le cadre où naguère on montrait toujours le pays" (Marcelin, 1913 : 13). L'épiscopat catholique est aussi contrarié par la progression numérique des cultes protestants en Haïti.

La campagne antisuperstitieuse est lancée alors qu'une circulaire présidentielle ordonne la répression du vodou. Cette décision de l'Exécutif survint dans

3. Le caractère "révolutionnaire " de la pensée indigéniste dans l'histoire intellectuelle et culturelle d'Haïti est discutée dans : Souffrant (1988), Trouillot (1993), Célius (2005: 71) et Métellus (2009: 7). L'œuvre de Price-Mars occupe une place considérable dans ces discussions parce qu'elle aura suscité un regard nouveau sur l'haïtianité et les croyances populaires haïtiennes. 
le contexte des assises criminelles qui se tenaient à Port-au-Prince. Parmi les cas en procès se distingue une affaire de meurtres commis par les nommés Soiriné François et Brévilus Saintilus. «Ce sont des cultivateurs. L'enfant de Soiriné vint à mourir ; l'on présuma que Argentine Jean-François, réputée comme sorcière et qui eut à soigner l'enfant, l'avait... mangé. De là, le crime, perpétré par nuit, par les deux accusés, sur l'habitation Decloche, en plaine du Cul-de-Sac. Argentine succomba des suites d'horribles blessures »(Anonyme, 1912a). La partie défenderesse nie la consistance des soi-disant preuves du crime et considère les accusés comme des victimes d'une erreur judiciaire. Elle critique également l'ordonnance de renvoi et l'acte d'accusation. Le jury, sous la présidence de Saint-Armand Lerebours, conclut finalement que les accusés sont non-coupables ${ }^{4}$. Quelques jours plus tard, probablement motivé par l'encombrante publicité de ce double procès en sorcellerie et de meurtres qui risque d'entacher la réputation d'Haïti à l'étranger, le président Cincinnatus Leconte adresse une circulaire aux commandants d'arrondissements :

«Général, le code pénal, vous ne devez pas l’ignorer, par ses articles 405, 406 et 407, interdit rigoureusement les pratiques du vaudoux et édicte les peines les plus sévères contre toute infraction à ses dispositions qui sont d'ordre public.

Or, il est de notoriété publique que les danses qui sont l'apanage ordinaire de ces manifestations de la superstition populaire, réunissent fréquemment les cultivateurs et cultivatrices tant dans les bourgs que dans les banlieues plus ou moins éloignées des villes; ces danses s'accompagnent elles-mêmes de toutes les pratiques habituelles de ce culte grossier. [...] Le Gouvernement, qui sait bien à quel point un tel état de choses tient à la condition arriérée et aux mœurs incultes de nos populations, n'entend vous prescrire dans cette circonstance aucun moyen violent. [...] Persuadez-vous, Général, qu'en tenant la main à ces injonctions, vous aiderez le Gouvernement dans la partie la plus efficace de son œuvre de l'élèvement national et de civilisation » (Leconte, $1912: 2$ ).

Le quotidien Le Matin, dans lequel publiait alors une pléiade d'intellectuels, rend hommage à la circulaire présidentielle du 23 mars 1912 par laquelle le président Cincinnatus Leconte fait défense formelle de « danser » le vodou. Elle y est perçue comme un signe de progrès civilisationnel vu qu'il s'agit de combattre les «superstitions populaires, particulièrement le vaudoux, dans la mesure où ces pratiques grossières portent atteinte à l'intérêt bien entendu des cultivateurs eux-mêmes et entretiennent l'oisiveté et tombent sous le coup de la loi pénale »

4. Il est important de signaler la fameuse affaire "Mercédès Fortuné ", du nom d'une jeune fille qui aurait été rituellement assassinée au Bel-Air par un ougan (prêtre du vodou) en 1903. L'affaire fut jugée en 1904 et, l'année suivante, le Tribunal de Cassation confirma la condamnation des principaux accusés, Saint-Elme Saint-Surin et sa mère Paulinette Hyppolite, à la peine capitale. Leurs six complices, à l'exception de Lucie Bazile, furent condamnés à deux ans d'emprisonnement. Le Nouvelliste, relatant l'affaire, écrit : "Ce jugement comporte son enseignement. Il nous apprend qu'il est plus que temps que l'on rompe avec ces croyances "moyenâgeuses" perturbatrices de tout progrès social sérieux, et que l'effort de tous, à quelque degré qu'ils appartiennent, doit converger vers ce noble but : relever le moral des victimes de l'ignorance par l'instruction et le bon exemple ». (Le Nouvelliste, 1905a). 
(Anonyme, 1912b). Dans Le Nouvelliste, un ancien député ne cache pas sa satisfaction à la suite de la publication de la circulaire :

"Il y a longtemps qu'on aurait dû y penser, car le Vaudoux a pris dans certains endroits, et notamment à la Baie-de-Henne, une extension telle que ses adeptes le placent bien au-dessus de la religion Catholique. - Tout pour le Vaudoux, rien sans le Vaudoux ! On peut compter à la Baie-de-Henne ceux qui ont le vaudoux en horreur et qui lui livrent une guerre à outrance afin d'enrayer dans ce petit coin de terre l'extrême corruption qu'il a implantée. [...] Autrefois on allait loin prendre ces ébats superstitieux, mais depuis qu'une autorité militaire est venue pour de l'argent leur permettre l'accès au bourg, c'est dans son sein même, à notre grand désespoir, que les adeptes du vaudoux s'adonnent passionnément à leurs cérémonies barbares " (Thomas, 1912).

À ce moment, alors inspecteur de l'Instruction Publique, Jean Price-Mars publie un article dans la revue bi-mensuelle Haïti Littéraire et Scientifique ${ }^{5}$ dans lequel il exprime ses positions par rapport à l'œuvre des Frères de l'Instruction Chrétienne (FIC) à la Grande Rivière du Nord. Tout en précisant qu'il n'est " inféodé à aucune confession religieuse », Price-Mars (1912 : 314) flatte l'initiative privée de l'abbé Darricades qui, à ses yeux, est un homme généreux doté d'un grand d'esprit. Ce dernier, confie l'auteur, a " choisi l'école pour auxiliaire » de son apostolat religieux. C'est aussi en ces termes élogieux que Price-Mars dresse le bilan de l'œuvre missionnaire de l'abbé Darricades :

«Le résultat, c'est que Bail y Bahon où il a rétabli l'ancienne chapelle, est devenu maintenant un centre plein d'activité. [...] Son école compte près de cent élèves; les mariages y sont devenus de plus en plus fréquents; de blanches et propres maisonnettes se dressent autour de son clocher. Bahon se civilise. Même le Vaudoux y a reculé avant la circulaire de M. le Président de la République. Tout cela est dû à l'activité de l'honorable M. Darricades. [...] Voilà bien une expérience qui a réussi : création de classes normales dans une école d'enseignement primaire qui fonctionne bien, pour la formation des maîtres de l'enseignement rural. Pour généraliser cette expérience, il faut d'abord s'assurer de la marche, dans un centre urbain de quelque importance, d'une école dont le personnel offre toutes les garanties de savoir et d'autorité. Dans l'état actuel de notre enseignement, je ne vois guère que les bonnes écoles congréganistes qui puissent réaliser ce double dessein; et si je pouvais aller jusqu'au fond de ma pensée, je confierais volontiers, tant à la campagne que dans les villes, tout notre enseignement primaire à la Congrégation des Frères de l'Instruction Chrétienne " (Price-Mars, 1912 : 314).

Ces propos, peu relatés dans la littérature ethnologique sur les productions de Price-Mars, n'ont rien d'étonnant. Né en 1876 à la Grande Rivière du Nord, dans une famille partagée par la piété d'un père protestant et le zèle d'une grandmère catholique, Price-Mars appartient à une génération d'intellectuels qui ont

5. Cette revue, dirigée par Edmond Laforest, avait Dantès Bellegarde pour rédacteur en chef et Abel Nicolas Léger pour secrétaire de la rédaction. Le premier est un francophile notoire. Bellegarde est un éminent intellectuel catholique qui, au début des années 1940, a collaboré avec le journal officiel du clergé catholique La Phalange. Abel N. Léger est un intellectuel qui s'est fait remarquer, tout au long de sa carrière diplomatique, par des œuvres de défense de la race noire et de l'image d'Haïti à l'étranger. 
vécu ou reçu l'influence du discours justificatif de la première campagne antisuperstitieuse (1896-1900) ${ }^{6}$. D'ailleurs, à cette occasion, les élites intellectuelles du département du Nord ont largement soutenu l'action de l'Église catholique pour exorciser le pays d'un «mal » qui fait honte à leur quête civilisatrice.

\section{L'influence durkheimienne sur la plume de Price-Mars}

Si aujourd'hui le vodou est élevé au rang de " religion nationale » et considéré comme matrice culturelle d'Haïti, c'est notamment dû à la réception positive du livre du docteur Jean Price-Mars en 1928: Ainsi parla l'Oncle ${ }^{7}$. Dans cet essai ethnographique sur le "folk-lore " haïtien, le savant médecin propose une approche particulière de la culture populaire haïtienne en s'inspirant notamment des travaux de plusieurs ethnologues coloniaux français sur l'Afrique ${ }^{8}$. Mais il lui revient le mérite d'avoir réhabilité le vodou haïtien en le présentant non plus comme un sujet de honte, mais comme une trace significative de l'africanité à l'origine de l'identité nationale ${ }^{9}$. Une telle lecture ne serait pas possible sans une démonstration de l'applicabilité du concept durkheimien de « religion » au culte vodou.

6. Price-Mars est à la fois une personnalité politique et un célèbre écrivain haïtien. Il a été député de la Grande Rivière du Nord (1905-1908) avant d'occuper des postes diplomatiques à Paris, Berlin, Washington et Santo Domingo. Sénateur (1930-1935), il ne réussit pas à se faire élire aux présidentielles de 1930. En 1941, il fut à l'origine de la fondation, en pleine campagne antisuperstitieuse (1939-1942), du Bureau d'Ethnologie d'Haïti et de l'Institut d'Ethnologie. Son engagement auprès $\mathrm{du}$ "monde noir " lui valut une reconnaissance mondiale. Depuis la parution d'Ainsi parla l'Oncle (1928), les écrivains de la " génération de la honte ", pour lui marquer leur respect, le surnomment «l'Oncle». En 1956, son jubilé lui attira les hommages des plus grands noms des études africanistes et des intellectuels haïtiens. Cette même année, il fut aussi élu, à l'unanimité, comme président du premier Congrès des Écrivains et Artistes Noirs et nommé premier président de la Société Africaine de Culture. Candidat au prix Nobel de littérature de 1959, il a récolté différents prix prestigieux et des titres honorifiques. L'Oncle aura publié, à sa mort en 1969 , une quinzaine d'essais et de très nombreux articles.

7. Je m'interroge sur le choix du titre de son livre qui rappelle étrangement un ouvrage d'Erckmann-Chatrian (deux écrivains français qui cosignaient leur ouvrage : Émile Erckmann et Alexandre Chatrian). Dans ce livre (Erckmann-Chatrian, 1867), il y a un petit "roman » titré « Dames Thérèse ou les volontaires de 92 » où, à trois reprises, on lit des passages commençant par : «Ainsi parla l'Oncle». Dans son conte intitulé «Le coquillage de l'oncle Bernard », Erckmann-Chatrian (1866:59) a aussi un passage débutant par "Ainsi parla l'Oncle ».

8. Citons notamment: Le Hérissé (1911) et Delafosse (1922). Rappelons qu'Ainsi parla l'Oncle est un "essai ethnographique " dont "l'ambition [est] de relever aux yeux du peuple haïtien la valeur de son folk-lore. Toute la matière de ce livre n'est qu'une tentative d'intégrer la pensée populaire haïtienne dans la discipline de l'ethnographie traditionnelle ». L'essai étudie notamment les "croyances populaires", le poids des traditions culturelles africaines dans le présent haïtien, l'animisme africain, etc.

9. En 1952, Price-Mars (1952: 7) définit ainsi l'identité nationale en se posant la question suivante : "Ce que nous sommes ? ". Il répond : «Des haïtiens. Je veux dire : un peuple qui, placé au centre de la méditerranée américaine dans la conjonction de courants divers de civilisation, ajoute à ces virtualités intrinsèques ce qu'il s'est assimilé de ses progéniteurs européens 


\section{Le vodou : objet du mépris intellectuel}

Jusqu'à la fin des années 1920, de multiples productions littéraires et d'ouvrages à prétention scientifique dénonçaient l'existence du vodou en Haïti. Elles le décrivaient généralement comme une "fête orgiastique (...) d'une nature démoniaque » (Alfred, 1873 : 349) durant laquelle les participants se livraient souvent à des pratiques de sorcellerie et de meurtres rituels ${ }^{10}$. Depuis l'affaire Claircine, du nom d'une fillette qui aurait été sacrifiée par un groupe de "sectateurs du vodou " en décembre 1863, c'était l'un des sujets de prédilection des essayistes et voyageurs étrangers traitant d'Haïti ${ }^{11}$. Cette affaire semble même à l'origine de certaines définitions du vodou. Par exemple, dans un dictionnaire encyclopédique $\mathrm{du}$ $\mathrm{XIX}^{\mathrm{e}}$ siècle, le vodou est présenté comme un :

«Culte des nègres aux Antilles. D'après quelques auteurs, le vaudou serait la plus étrange des superstitions que l'Afrique ait importées en Amérique et particulièrement à Haïti. [...] Les jours de grande cérémonie, on tue une poule noire et un cabri, dont les affiliés boivent le sang; après quoi la foule se livre à mille contorsions extatiques et pousse des cris effroyables. On prétend que ces fêtes sont terminées par le sacrifice de petits enfants dont les prêtres se partagent les membres sanglants dans un horrible repas" (Trousset, s.d. : 581).

Face à cette situation, les élites intellectuelles affichaient différentes attitudes. En 1883, par exemple, l'idéologue protestant Louis-Joseph Janvier indiquait la disparition graduelle du vodou dans les campagnes haïtiennes. Dans une polémique engagée avec Victor Cochinat, il affirmait que « toutes les vieilles danses africaines : le banda, le madouka, l'arada, le congo, le séba, l'ibo, etc. ont complètement disparu aussi bien dans les villes que des campagnes. [...] Il y a là un rapide travail de transformation sociale, toute une série de répudiations tacites et d'aspirations nouvelles, mais élevées, vigoureuses et nobles et qui sont pour être encouragées » (Janvier, 1883 : 94). Pour Bénito Sylvain (1894: 4), grand artisan haïtien du panafricanisme et alors président du Comité oriental et africain de la Société d'Ethnographie de Paris, «le nombre est grand des Haïtiens qui, comme moi, ignoraient totalement, avant de venir à Paris, en quoi consistait au juste le

et africains - qualités et défauts - afin de forger son propre destin (sic) ". Dans une autre conférence, prononcée à la Sorbonne le 12 mars 1959 alors qu'il était ambassadeur d'Haïti en France, Price-Mars (1971 : 57) attire l'attention de l'Association Internationale pour la Culture Française à l'Étranger sur la nature de l'identité haïtienne : "Nous ne sommes ni les "français colorés" dont se gargarisent les attardés d'un colonialisme suranné, ni les africains dont se réclament des racistes à rebours $($ sic $) »$.

10. «Il n'est que trop certain que le cérémonial du Vaudoux est, actuellement encore, accompagné, à Haïti tout au moins, de sacrifices humains et d'actes de cannibalisme. Sir Spencer assista lui-même un jour au procès de sectataires du Vaudoux accusés et convaincus d'avoir tué et mangé un enfant. Et cette affreuse fringale de chair humaine ne s'exerce pas seulement sur les victimes immolées au dieu-serpent, mais bien encore en dehors de toute cérémonie soi-disant religieuse, simplement pour satisfaire un appétit dépravé : des parents mangeant les corps des leurs, des mères même dévorant leurs propres enfants!» (L. D., 1886:343).

11. Signalons notamment: Saint-John (1886), Texier (1891), Prichard (1900) et Caplain (1904). 
Vaudoux. C'est la lecture des charitables journaux français qui a fini par nous renseigner là-dessus. On nous dit que c'est le culte de la couleuvre... Ne serait-ce pas une couleuvre qu'on voudrait faire avaler en Europe ? "D'autres intellectuels haïtiens, évitant la thèse d'un vodou en voie de disparition, préféraient attirer l'attention de leurs détracteurs sur le fait que, sous des formes diverses, les "superstitions » existaient partout. Il était clair que, à cette époque, parler du vodou constituait une honte pour les intellectuels haïtiens. C'est peut-être une raison expliquant que le vodou soit si peu étudié par ces derniers jusqu'aux années 1930.

\section{La dimension durkheimienne de la pensée de Price-Mars}

Durant l'Occupation américaine d'Haïti (1915-1934), Price-Mars entame une série de conférences dans l'espoir de susciter l'élan patriotique des élites nationales. C'est dans ce contexte qu'il publiait déjà ses réflexions sur La Vocation de l'élite (Price-Mars, 1919) pour dénoncer le bovarysme collectif et la francophilie des élites haïtiennes. Découvrant la notion de «folk-lore » et sa promotion dans les sciences sociales, Price-Mars croit tenir une piste pour exprimer davantage son patriotisme. Dans une conférence prononcée en 1952, il défend l'idée qu'il est bien placé, en qualité d'Haïtien ${ }^{12}$, pour défendre l'image de son pays et les liens indissolubles entre le patriotisme et le «folk-lore » qui doit être nécessairement valorisé :

«Le folk-lore étant l'expression d'un fait humain, notre folk-lore à nous est la manifestation de croyances et de mœurs qu'on est susceptible de rencontrer selon des modalités diverses n'importe où sur la surface du globe. Par conséquent, il ne saurait y avoir d'indignité ou d'incorrection à en dépister l'existence, à en examiner les avatars, à en révéler les possibilités artistiques. C'est encore l'un des aspects de la connaissance auquel mes amis et moi nous nous sommes consacrés dans la plénitude de nos droits et de nos devoirs d'authentiques haïtiens. Nous en revendiquons la fierté et l'honneur » (Price-Mars, 1952 : 16).

Cet aveu tardif de l'auteur permet de proposer une autre lecture de son chefd'œuvre: Ainsi parla l'Oncle. La rédaction de cet ouvrage, sans même s'inscrire dans un cadre clairement polémique, est surtout motivée par une volonté de défendre l'image d'Haïti face à ces détracteurs de l'étranger. Avant 1928, rares sont les intellectuels haïtiens à traiter du vodou de crainte que cela ne ternisse davantage l'image d'Haïti parmi les élites occidentales ${ }^{13}$. Peu d'écrivains et

12. Price-Mars pense, dans ce contexte, à une polémique qu'il eut avec le Père Joseph Foisset, érudit du clergé breton en Haïti, sur la pertinence scientifique d'Ainsi parla l'Oncle. Il avoue que "défendre le patriotisme n'est vraiment un acte inestimable que lorsqu'on est en mesure de s'appuyer sur les droits imprescriptibles que vous confère votre nationalité. Hors de ce critère, il est pour le moins singulier qu'un étranger puisse professer des leçons de patriotisme aux habitants d'un pays qui n'est pas le sien si louables et si sincères que puissent être ses intentions" (Price-Mars, $1952: 8)$.

13. Trouillot (1885), Lhérisson (1899, 1901), Légitime (s.d.), Vincent (1910), Eddah (1905a, 1905b, 1905c, 1905d), Holly (1918). 
d'artistes ont placé le vodou au centre de leurs créations ${ }^{14}$. Une des contributions majeures d'Ainsi parla l'Oncle est d'avoir élaboré une approche scientifique permettant d'aborder le vodou sans faire rougir les élites haïtiennes.

En fait, contrairement au discours catholique officiel, son approche considère le vodou comme "un vrai culte populaire " (Price-Mars, 1962:146) et non comme de simples "pratiques superstitieuses ». Price-Mars précisera, plus tard, qu'une remise en question de la notion de "superstition » est toujours nécessaire pour appréhender le vodou et rester dans l'objectivité scientifique. Il plaide ainsi pour une méthode se distanciant des étiquettes négatives collées sur l'objet d'étude et contre l'usage de certaines notions confuses couramment utilisées dans les sciences des religions. Il définit la superstition comme une notion dont le contenu est relatif.

" C'est un sentiment subjectif qu'on applique aux croyances que nous jugeons indignes de nous, parce que nous nous considérons comme trop sages et trop avancés pour nous y attacher. Cependant, le sceptique qualifie de superstition la croyance aux miracles, le chrétien considère comme superstition la révérence de l'indou devant un animal. Que de points de vue. Donc le vodou est une religion comme une autre, mais elle est la religion des masses, l'élite se considérerait dégradée, si ouvertement elle affectait d'y croire " (Price-Mars, 1962 : 146-147).

Dans Une étape de l'évolution haïtienne, essai de 1929, Price-Mars écrit que le vodou repose "sur un fond de magie et de superstition".

"Nous ne l'avons jamais considéré que sous cet angle et c'est pourquoi il est devenu synonyme d'ésotérisme. En réalité, il n'a pas échappé à la loi qui semble régir la vie des religions. La superstition et la magie l'ont envahi comme la gangue s'attache au pur métal. Car, vous savez, la magie n'est que la contrefaçon de la religion, la superstition n'en est que la déformation ou la caricature. [...] Quoi qu'il en soit, une autre question se pose. Les nègres de Saint-Domingue ont-ils été les grossiers superstitieux dont nous parlent nos historiens ? [...] Il n'est pas de superstition capable d'engendrer le souffle magnifique de spiritualité qui souleva les âmes de nos pères et conditionna le miracle de 1804. Superstition, la foi qui poussa les bandes, sans armes, d'Hyacinthe en 1791, dans la plaine du Cul-de-Sac, à se ruer sur l'artillerie des bataillons d'Artois et à plonger leurs mains dans la gueule des canons pour empêcher les boulets d'en sortir ?" (Price-Mars, 1929 : 145-146).

Cette parenthèse permet de préciser que, dans la pensée de Price-Mars, toute religion suppose une caricature superstitieuse. Il passe en revue, par exemple, l'attitude de certains intellectuels catholiques s'attachant aux symboles de l'Église. Il en profite aussi pour rappeler qu'Haïti n'a pas le monopole de la superstition. En Bretagne, souligne-t-il, "quand le prêtre a béni dans un grand bassin de cuivre placé au milieu de l'église l'eau qui doit être versée toute l'année dans les

14. Rappelons un fait peu connu. En mai 1905, sous la direction du maestro Occide Jeanty, la Musique du Palais National a fait applaudir sa "Méringue Caractéristique, très intéressante par son rythme bizarre d'une danse de vaudou " (Le Nouvelliste, 1905b). À noter les romans de mœurs locales de Frédéric Marcelin (1902) et d'Antoine Innocent (1906) qui décrivent, sans ambages, l'ancrage du vodou en milieu rural haïtien. 
bénitiers, les bonnes femmes se bousculent, se battent même pour arriver les premières à remplir les petites bouteilles qu'elles ont apportées, persuadées que celles qui y parviendront, prenant la crème seront plus favorisées que les autres, et surtout que le lait de leurs vaches sera plus abondant » (Price-Mars, 1929 : 148-149). Mais comment Price-Mars démontre-t-il que le vodou est une religion ? En quoi est-il redevable à Durkheim ?

Pour répondre à ces interrogations, rappelons d'abord comment Durkheim construit le concept de "religion ». Le sociologue s'est intéressé aux " attitudes rituelles qui, malgré la diversité des formes que les unes et les autres ont pu revêtir, ont partout la même signification objective et remplissent partout les mêmes fonctions. Ce sont ces éléments permanents qui constituent ce qu'il y a d'éternel et d'humain dans la religion » (Durkheim, 1912: 6). Dans les Formes, il projette d'étudier «la religion la plus primitive et la plus simple qui soit actuellement connue, d'en faire l'analyse et d'en tenter l'explication " (Durkheim, 1912 : 1). Ici, l'idée de primitivité renvoie à un système religieux "qui se rencontre dans des sociétés dont l'organisation n'est dépassée par aucune autre en simplicité ; il faut de plus qu'il soit possible de l'expliquer sans faire intervenir aucun élément emprunté à une religion antérieure » (Durkheim, 1912:1).

Durkheim s'attaque aussi à des préjugés qui constituent un obstacle à l'appréhension objective du système totémique australien. En passant en revue différentes définitions de la religion, il se convainc qu'une religion peut exister indépendamment de toute référence à une divinité transcendante ${ }^{15}$. Ceci remet en cause toute une tradition ethnologique qui définit la religion par la croyance en des êtres spirituels, transcendantaux, avec lesquels l'homme croit pouvoir entrer en contact par des rituels variables (prières, sacrifices, rites propitiatoires, etc.). Cette croyance aux âmes et aux esprits a été longuement théorisée par Edward B. Tylor (1832-1917) qui en fait la première phase de la religion en tentant de démontrer que le "primitif " se rend compte de l'existence d'un principe différent de son corps à la suite d'expériences psychophysiologiques que sont les rêves, la mort, le sommeil, la maladie, la transe, etc. (Tylor, 1873). Et c'est même cette croyance qui a fait naître le culte des ancêtres dans les communautés pour lesquelles l'âme survit à la mort de l'individu. Que des esprits puissent se manifester dans les corps, les posséder, trouve aisément une explication dans le système animiste. Car les esprits jouiraient d'une existence autonome et de la capacité d'intervenir dans le monde objectif en présidant aux différents éléments de la nature. De là naquit le polythéisme des "peuples semi-civilisés ». La théorie de Tylor connut un grand succès dans les sciences sociales. En 1897, le professeur

15. Pour l'auteur, la notion même de "divinité » est floue et n'intègre pas nécessairement de nombreux esprits auxquels sont voués des cultes. C'est ce qui explique la substitution courante de ce terme par " être spirituel ». Tylor l'avait également remarqué et souligné qu'adopter une définition de la religion suggérant un culte adressé à un Être suprême risque de ne pas prendre en considération la vie religieuse de nombre de tribus connues (Durkheim, 1912: 40). 
Ludwig Stein de l'Université de Berne reconnaissait que les théories dominantes concernant la genèse de la religion étaient l'animisme de Tylor et de Lippert, la théorie du rêve et des esprits de Spencer, le fétichisme de Comte et de ses successeurs ainsi que le totémisme de Gruppe (Stein, 1897 : 4).

Mais, pour Durkheim (1912 : 41), les lectures de Tylor et de Frazer tendent à exclure nombre de formes religieuses où l'idée d'une divinité est absente ou ne joue qu'un rôle secondaire et effacé. Selon lui, les croyances et les rites occupent une place centrale dans l'expérience religieuse. En analysant les croyances religieuses, le sociologue découvre que celles-ci supposent une "classification des choses réelles ou idéales, que se représentent les hommes, en deux classes, en deux genres opposés, désignés généralement par deux termes distincts que traduisent assez bien les mots de profane et de sacré »(Durkheim, 1912: 50). Au fil de cette phase de théorisation, il développe une approche fonctionnelle de la religion selon laquelle il s'agit d' " un système solidaire de croyances et de pratiques relatives à des choses sacrées, c'est-à-dire séparées, interdites, croyances et pratiques qui unissent en une même communauté morale, appelée Église, tous ceux qui y adhèrent» (Durkheim, 1912: 65).

S'inspirant du philosophe Salomon Reinach (1909), Price-Mars entame sa réflexion en exprimant un doute fondamental sur la pertinence empirique de l'étymologie latine (religio, religare) couramment évoquée pour définir le concept de religion. Cette étymologie orienterait vers la conclusion que le fait religieux doit nécessairement mettre en relation une divinité et des hommes. Mais une telle position ne risque-t-elle pas d'exclure d'emblée le bouddhisme dont le système de croyances ne repose pas sur un dieu ? Face à l'impératif de définir la « religion ", Price-Mars recourt à la théorie durkheimienne de la religion qu'il a découverte probablement durant ses études intermittentes ou sa carrière diplomatique en France ${ }^{16}$.

Price-Mars reproduit de longs extraits du sociologue français pour étayer ses propres thèses. Certaines fois, le lecteur avisé se demande même s'il est en train de lire une idée originale de Price-Mars ou la simple reproduction d'un passage de Durkheim. Par exemple, quand le sociologue français traite du bouddhisme, il fait état d'une série de points de vue d'auteurs sur ce thème (Barth, Oldenberg et Burnouf). Price-Mars les reprend à son compte sans fournir plus de détails. Citant Durkheim sans insister néanmoins sur sa démonstration, Price-Mars (1928: 29-30) écrit ainsi :

16. En 1899, grâce à une bourse du gouvernement haitien, Price-Mars se rendit en France pour des études de médecine. C'est là, en fréquentant la Sorbonne, le Collège de France et le Musée de Trocadéro, qu'il développe un intérêt pour les sciences sociales. Il sera, de 1915 à 1916, ministre plénipotentiaire d'Haïti à Paris. Notons que Price-Mars cite seulement deux fois le nom de Durkheim dans le corps de son texte. Néanmoins, dans sa polémique avec le Père Foisset en 1945, il exprime toute sa dette envers le sociologue français. Sur cette polémique, voir : Clorméus (2012). 
«L'ethnographie et l'histoire semblent nous donner raison. N'existe-t-il pas de grandes religions d'où l'idée de dieux et d'esprits est absente où, tout au moins, elle ne joue qu'un rôle secondaire et effacé ? C'est le cas du Bouddhisme notamment. Le bouddhisme, dit Burnouf, se place en opposition au brahmanisme comme une morale sans dieu et un athéisme sans nature. "Il ne reconnaît point de dieu dont l'homme dépende, dit M. Barth". Sa doctrine est absolument athée et M. Oldenberg, de son côté, l'appelle "une religion sans dieu". [...] On a voulu faire ressortir simplement que si une grande religion comme le bouddhisme a pu naître et vivre pendant un certain temps dans sa pureté originelle d'après un concept tout à fait laïque, la définition donnée ci-dessus de la religion, à savoir qu'elle est un lien entre la divinité et l'homme, exclurait le bouddhisme du cadre des religions et que cette conclusion serait paradoxale. Donc nous éliminerons comme étant trop caractéristique des religions déjà parvenues à un terme d'une haute évolution, l'acceptation qui en fait le symbole d'un rattachement de l'homme à un être ou à des êtres spirituels dont il dépend ».

Ceci dit, Price-Mars (1928 : 30) relève ce qui lui paraît essentiel dans l'approche durkheimienne de la religion :

"L'idée adoptée par l'école sociologique de Durkheim contient la pensée minima que nous recherchons. Elle établit, et tout le monde est d'accord là-dessus, que "toutes les croyances religieuses connues, qu'elles soient simples ou complexes, présentent un même caractère commun : elles supposent une classification des choses réelles ou idéales que se représentent les hommes en deux genres opposés, désignés généralement par deux termes distincts que traduisent les mots de profane et de sacré. La division du monde en deux domaines comprenant l'un, tout ce qui est sacré, l'autre, tout ce qui est profane, tel est le trait distinctif de la pensée religieuse ; les croyances, les mythes, les dogmes, les légendes sont ou des représentations ou des systèmes de représentations qui expriment la nature des choses, les vertus et les pouvoirs qui leur sont attribués, leur histoire, leurs rapports les unes avec les autres et avec les choses profanes”. [...] En résumé, le sacré et le profane forment deux catégories distinctes dont le signe différent réside dans le caractère opposé et absolu de l'une et de l'autre catégories ».

Évidemment, Price-Mars n'ignore pas que la plupart des religions reposent sur des croyances en une ou plusieurs déités auxquelles s'adressent des cultes particuliers. D'ailleurs, ne cite-t-il pas les réflexions de l'abbé Joseph Bricout (1867-1930 ${ }^{17}$ qui estime que "partout la religion suppose la croyance à des êtres supérieurs, tels, du moins, que l'homme en dépende et ait quelque chose à en craindre ou à en espérer, qu'il puisse appeler à son aide, dont il puisse s'assurer le concours » (Bricout, 1911 : 15 ; Price-Mars, 1928 : 30). Dans cette perspective, fort de ce qu'il retient du livre de Durkheim, Price-Mars (1928: 32) aboutit à la conclusion que le vodou est une religion comme toutes les autres :

«Le Vaudou est une religion parce que tous les adeptes croient en l'existence des êtres spirituels qui vivent quelque part dans l'univers en étroite intimité avec les humains dont ils dominent l'activité. [...] Le Vaudou est une religion parce que le culte dévolu à ses dieux réclame un corps sacerdotal hiérarchisé, une société de fidèles, des temples, des autels, des cérémonies et, enfin, toute une tradition orale qui n'est certes pas parvenue jusqu'à nous sans altération, mais grâce à laquelle se transmettent les parties essentielles de ce culte.

17. L’abbé Joseph Bricout a été directeur de la Revue du Clergé Français. Ancien vicaire à Clignancourt, ami et élève du Père Alfred Loisy, il s'intéressait particulièrement aux questions d’apologétique chrétienne et du surnaturel. 
Le Vaudou est une religion parce que, à travers le fatras des légendes et la corruption des fables, on peut démêler une théologie, un système de représentation grâce auquel, primitivement, nos ancêtres africains s'expliquaient les phénomènes naturels et qui gisent de façon latente à la base des croyances anarchiques sur lesquelles repose le catholicisme hybride de nos masses populaires".

Tout le problème est alors de préciser quelle est la valeur morale du vodou. D'emblée, Price-Mars reconnaît que « toutes les religions ont leur morale et que celle-ci est le plus souvent en relation étroite avec l'évolution mentale du groupe où cette religion a pris naissance et s'est enracinée » (Price-Mars, 1928 : 32-33). Concernant la religion populaire haïtienne, il constate que les adhérents se soumettent à une série d'interdits et de contraintes qui constitue " un code de tabous ». Alors le vodou ne dispose-t-il pas d'une morale propre ? Price-Mars (1928 : 35) estime que :

«Il ne paraît en être dénué que parce que, malgré nous, nous le jugeons en fonction d'un type de morale plus élevée, adéquate à notre conception de la vie, parce qu'enfin nous jugeons la morale du Vaudou comme une superstition injurieuse pour notre idéal de civilisation. Que si, au lieu de la considérer en comparaison de la morale chrétienne, on la jugeait à sa valeur intrinsèque, on verrait par la sévérité des sanctions auxquelles s'expose l'adepte qui transgresse "la loi", combien celle-ci commande une discipline de la vie privée et une conception de l'ordre social qui ne manquent ni de sens ni d'à-propos. On comprendrait ainsi comment, à un moment donné, elle fut assez efficace pour brider les instincts de l'individu dans une certaine mesure et préserver la dissolution de la communauté ».

Évidemment, une telle démonstration ne passe guère inaperçue dans la presse et la communauté scientifique de Port-au-Prince qui lui consacrent de nombreux comptes rendus. En dépit de quelques reproches, Ainsi parla l'Oncle est reçu positivement par le réseau de jeunes étudiants et d'activistes politiques engagés dans le mouvement anticlérical enclenché par des leaders protestants ${ }^{18}$. Cette œuvre libère davantage la parole et indique une voie à une quête identitaire aiguillonnée par un anti-américanisme triomphant en Haïti et un déficit d'actualisation de la francophilie ${ }^{19}$ dans un contexte de changement culturel à l'échelle

18. Il faut souligner que le but de cette campagne anticléricale n'était pas de favoriser les cultes réformés en Haïti. Il s'agissait de réclamer un clergé catholique indigène dans un contexte où les prêtres français desservant les paroisses d'Haïti étaient généralement perçus comme de précieux collaborateurs de l'Occupant américain. La nationalisation du clergé catholique survint, dans des conditions très difficiles, durant la présidence de François Duvalier (1957-1971). Voir : Duvalier (1968) et Arthus (2011).

19. La francophilie des élites haïtiennes date des premiers moments de l'histoire nationale. Dans leur quête d'organisation de la société, ces élites adoptèrent le modèle social français qui devait leur permettre, entre autres, de prétendre à la « civilisation ». Cette francophilie contribua à distinguer ces élites des " masses " paysannes qui perpétuaient des traditions culturelles héritées d'Afrique. L’Occupation américaine (1915-1934) légitima un discours politique visant à concilier ces deux entités sociales en vue de combattre l'ennemi commun. Il fallait définir, de ce fait, une identité culturelle susceptible d'afficher cette unité possible entre les élites et les masses rurales et indicatrice de la concrétisation du vouloir-vivre ensemble. Ce choix politicoidéologique délégitima progressivement la francophilie tout en attisant le sentiment " antiyankee ». 
internationale ${ }^{20}$. Le livre est aussitôt récupéré par les écrivains de l'école indigène, rebaptisée "mouvement indigéniste ", et, peu après, ceux de l'école des Griots qui vont se mettre à exalter l'Afrique. C'est dans ce contexte que prend naissance le roman paysan haïtien qui met en exergue l'ancrage et l'importance du vodou dans les communautés rurales ${ }^{21}$. Dans les années 1930, Ainsi parla l'Oncle circule dans les milieux estudiantins de Paris et tombe entre les mains de Léopold Sédhar Senghor, Léon Gontran Damas et Aimé Césaire qui s'en serviront pour constituer les bases des discours sur la négritude.

\section{Conclusion}

Pour sa contribution significative à l'étude et la valorisation de la culture haïtienne, Roger Bastide (1968 : 75) considère Ainsi parla l'Oncle « comme point de départ de toute la recherche afro-haïtienne et comme première définition valable du Vaudou ». Définition à laquelle se réfèrera le clergé concordataire, dès les années 1950, pour réajuster sa pastorale et ses stratégies missionnaires ${ }^{22}$. Mais, bien avant l'Église catholique, certaines figures du secteur culturel haïtien avaient déjà porté un regard positif sur le vodou. Werner Anton Jaegerhuber (1900-1953), en s'intéressant à l'étude du «folk-lore » initiée par Price-Mars, composa une "messe folklorique" et diffusa, en les réécrivant, des «chants vodou " jusqu'auprès des élites du pays. Avant son investiture présidentielle, au Carlton Hôtel (États-Unis d'Amérique), Élie Lescot assista à une représentation de chorégraphies yanvalou particulièrement marquées par la performance

20. Rappelons simplement qu'à Cuba et au Brésil, dès le début du XXe siècle, s'inaugurent des travaux sur l'afro-américanisme. Aux États-Unis, un sociologue d'origine haïtienne, William Edward Burghardt Du Bois (1868-1963), diplômé de Harvard, publie un ensemble de travaux sur la condition noire. En 1910, il est à l'origine de la création de la NAACP (National Association for the Advancement of Coloured People). Le premier congrès panafricain eut lieu à Paris en 1919 et le second à Londres et à Paris en 1921 (l'année où René Maran obtient le prix Goncourt pour son roman Batouala qui critique le système colonial). En 1928, l'ultranationaliste Marcus Mosiah Garvey (1887-1940) affirmait, lors d'une conférence à Paris, que les noirs devaient être fiers de leur "race » et que l'Afrique était la terre promise, la «terre sans mal ». Cette même année, l'écrivain brésilien José Oswald de Souza Andrade (1890-1954) publie son Manifesto Antropófago.

21. C'est Jacques Roumain, écrivain haïtien d'inspiration marxiste, qui fut à l'origine de cette consécration en publiant La montagne ensorcelée (1931) et Les gouverneurs de la rosée (1944).

22. En 1955, Monseigneur Paul Robert, l'un des grands artisans de la troisième et dernière campagne antisuperstitieuse (1939-1942), reconnaît la valeur de la démonstration de PriceMars en admettant que le vodou est une religion. Dans une plaquette destinée au service du ministère apostolique, l'évêque écrit : "On peut facilement constater en effet que le Vaudou contient les éléments fondamentaux d'une religion : une croyance profonde à des divinités, et cette foi va jusqu'au fanatisme; le désir d'entrer en communication avec ces divinités jusqu'à la possession; des cérémonies par lesquelles on cherche à apaiser leur colère ou obtenir leur faveur ; un sacerdoce hiérarchisé, organisant le culte, présidant les cérémonies, intermédiaire entre les fidèles et les dieux, habilité pour manifester aux fidèles les communications de la divinité, prescrire les ordonnances religieuses et offrir les sacrifices » (Robert, $1955: 3-4$ ). 
artistique de Léon Destiné et Gladys Hippolyte (Anonyme, 1941b), en présence d'importantes personnalités politiques ${ }^{23}$. On comptait dans l'assistance : Fernand Dennis (ministre haïtien des Affaires étrangères), les ambassadeurs du Pérou, du Brésil, du Chili, de Cuba, de l’Équateur, etc. (Macnair, 1941). La soprano Andrée Lescot, fille du président Élie Lescot (1941-1946), s'illustra en interprétant, dans un documentaire pour une institution canadienne, trois chants du répertoire folklorique haïtien inspirés du vodou ${ }^{24}$.

Ce tournant dans l'histoire culturelle d'Haïti est certainement dû en grande partie au charisme et à l'œuvre intellectuelle du docteur Jean Price-Mars. Mais, malheureusement, les études scientifiques insistent peu sur ses référents théoriques et ses conceptions épistémologiques. Il est indéniable que, pour valoriser le « folk-lore » haïtien, il a puisé des arguments dans les travaux d'auteurs nationaux et étrangers. En ce qui concerne particulièrement la réhabilitation du vodou, telle qu'engagée dans Ainsi parla l'Oncle, c'est surtout à Émile Durkheim qu'il doit sa démonstration.

Lewis Ampidu CLORMÉUS Centre d'Études Interdisciplinaires des Faits Religieux (EHESS-CNRS) lclormeus@yahoo.fr

\section{Bibliographie}

ANONYME, 1912a (14 mars), " Assises criminelles. Sorcellerie et meurtre ", Le Nouvelliste, $\mathrm{n}^{\circ} 4073$, p. 2.

-, 1912b (28 mars), "À propos de deux circulaires présidentielles ", Le Matin, n 1509, p. 1.

-, 1941a (6 mai), "Au son des tambours les Haïtiens chantent les louanges aux Dieux vaudouesques ", Le Matin, $\mathrm{n}^{\circ} 10308$, p. 2, 5.

-, 1941b (8 mai), "Le groupe folklorique haïtien aux États-Unis ", Le Nouvelliste, $\mathrm{n}^{\circ} 20851$, p. 1.

-, 1941c (11-12 mai), «Le grand succès de la Huitième fête annuelle du Folklore National à Washington ", Le Matin, n 10313, p. 1.

23. Ces artistes haïtiens, conduits par Lina Mathon (1902-1993), étaient invités à participer au huitième Festival annuel de folk-lore qui se tint aux États-Unis d'Amérique ( $1^{\text {er }}-3$ mai 1941) au cours duquel se produisirent au moins 500 acteurs à Constitution Hall. Le Matin fut choqué de leur prestation au banquet du Carlton Hôtel et leur reprocha leur manque de professionnalisme, les crises de possession répétées sur scène, etc. De plus, l'auteur anonyme de l'article prend la précaution de préciser qu' "on a expliqué que les Haïtiens ne croient pas au Vaudouisme. Ce n'est qu'une danse qu'ils aiment exécuter de temps à autre " (Anonyme, 1941a). Pourtant, quelques jours plus tard, Le Matin reproduit, à la première page de son double numéro des 11-12 mai 1941, les propos enthousiastes du Washington Post soulignant que ces artistes haïtiens ont particulièrement retenu l'attention (Anonyme, 1941c).

24. Talents Canadiens, numéro 3. Chansons Créoles avec Andrée Lescot. Film documentaire réalisé par Roger Blais de l'Office National du Film, 1952. 
ARTHus Wien Weibert, 2011, "Les relations internationales d'Haïti (1957-1971) : la politique étrangère de François Duvalier ", Thèse de doctorat, Université Paris I Panthéon-Sorbonne.

Aubin Eugène, 1910, En Haïti. Planteurs d'autrefois. Nègres d'aujourd'hui, Paris, Librairie Armand Colin.

Bastide Roger, 1968, "Nécrologie. Fernando Ortiz. Jean Price-Mars ", Journal de la Société des Américanistes, tome 57, p. 73-75.

BRICOUT J. (coord.), 1911, Où en est l'histoire des religions ?, tome 1 : Les religions non chrétiennes, Paris, Letouzey et Ané Éditeurs.

Caplain Jules, 1904, La France en Haïti. Catholicisme, Vaudoux, Maçonnerie, Paris, Imprimerie F. Levé.

Célıus Carlo Avierl, 2005, "La création plastique et le tournant ethnologique en Haïti ", Gradhiva, no 1, p. 71-94.

Clorméus Lewis Ampidu, 2012, Entre l'État, les intellectuels et les religions. Redécouvrir la campagne anti-superstitieuse de 1939-1942, Thèse de doctorat, École des hautes études en sciences sociales/Université d'État d'Haiti.

Delafosse Maurice, 1922, Les Noirs de l'Afrique, Paris, Payot et Cie.

Demolins Edmond, 1897, À quoi tient la supériorité des Anglo-Saxons, Paris, Librairie de Paris/Firmin-Didot et Cie, Imprimeurs-Éditeurs.

Dévot Justin, 1905 (25 janvier), «Memoranda », Le Nouvelliste, nº 1930, p. 2.

DurкheIM Émile, 1893, De la division du travail social. Thèse présentée à la Faculté des Lettres de Paris, Paris, Félix Alcan Éditeur.

-, 1912, Les formes élémentaires de la vie religieuse. Le système totémique en Australie, Paris, Librairie Félix Alcan.

Duvalier François, 1968, Mémoires d'un leader du Tiers-Monde, Paris, Éditions Hachette. EDDAH, 1905a (12 septembre), "Le Vaudoux ", Le Nouvelliste, no 2119, p. 1-2.

-, 1905b (28 septembre), "Le Vaudoux ", Le Nouvelliste, $\mathrm{n}^{\circ} 2133, \mathrm{p} .1-2$.

-, 1905c (16 octobre), "Le Vaudoux », Le Nouvelliste, $n^{\circ}$ 2146, p. 1-2.

-, 1905d (9 novembre), " Ma dernière au Vaudoux ", Le Nouvelliste, no 2165, p. 1-2.

ERCKMANN-Chatrian, 1866, Contes populaires, s.l.

-, 1867, Romans nationaux, Paris, J. Hetzel Éditeurs.

Holly Arthur, 1918, Les daïmons du culte voudo, Port-au-Prince, Imp. Edmond Chenet. InNocent Antoine, 1906, Mimola, Port-au-Prince, Imprimerie F. Malval.

JANVIER Louis-Joseph, 1883, La République d'Haïti et ses visiteurs (1840-1882). Réponse à M. Victor Cochinat (de la Petite Presse) et à quelques écrivains, Paris, Marpon et Flammarion, Libraires-Éditeurs.

L. D., 1886 (août), « Haïti ou la République Noire, par sir Spencer St. John, ancien ministre résident et consul général à Haïti. Traduction de l'anglais par M. J. West, capitaine de frégate en retraite. Paris, Plon, 1886 ; in-12, avec carte », Revue Maritime et Coloniale, tome 90, p. 342-343.

Le HérisSÉ Auguste, 1911, L’Ancien royaume du Dahomey. Mœurs, religion, histoire, Paris, Émile Larose Libraire-Éditeur.

Le Nouvelliste, 1905a (19 janvier), no 1925, p. 2.

-, 1905b (3 mai), nº 2010, p. 2.

,- 1905 c (27 octobre), no 2156, p. 2.

LECONTE Cincinnatus, 1912 (26 mars), " Pour l'Ordre Rural. Circulaire Présidentielle aux Commandants d'arrondissements ", Le Nouvelliste, n 4083, p. 2. 
LÉGITIME François-Denis, s.d., La vérité sur le vaudoux, Port-au-Prince.

LHÉRISSON Élie (Dr), 1899 (20 mars), «Du vaudou : Étude de quelques manifestations psychiques et somatiques observées chez les danseuses ", La Lanterne Médicale, vol. 2, n 3, p. 19-23.

-, 1901 (février), "Des démonomanes Vin-Bindingue ", La Lanterne Médicale.

MaCNAIR Marie, 1941 (4-5 mai), "Lescot reçoit avec des danseurs du vaudou avant de partir pour occuper la présidence d'Haïti ", Le Matin, n 10307, p. 2.

Marcelin Frédéric, La vengeance de Mama, Paris, P. Ollendorff.

-, 1913, Au gré du souvenir, Paris, Augustin Challamel Éditeur.

MAURY Alfred, 1873 (juin), « Die Rechtsverhältnisse bei verschiedenen Völkern der Erde, ein Beitrag zur vergleichenden Ethnologie, von Prof. Dr Bastian. Berlon, 1872. Le droit comparé des différents peuples de la terre, essai pour servir à l'ethnologie comparée, par le professeur Dr Bastian. Berlin, 1872, gr. in-8. ", Journal des savants, p. 344-355.

Métellus Jean, 2009, "Préface ", in Fleurimond Wiener Kerns, Haïti de la crise à l'occupation. Histoire d'un chaos (2004-2005), t. 2 : Les technocrates au pouvoir, Paris, L'Harmattan, p. 7-13.

Porsenna Fernand, 1905 (31 octobre), «À l’École de Droit ", Le Nouvelliste, no 2159, p. $1-2$.

Price-Mars Jean, 1912 (5 juillet), "La Réforme de l'Enseignement primaire », Haïti Littéraire et Scientifique, p. 312-317.

-, 1919, La vocation de l'élite, Port-au-Prince, Imprimerie Edmond Chenet.

-, 1928, Ainsi parla l'Oncle... Essais d'ethnographie, Compiègne, Imprimerie de Compiègne.

-, 1929, Une étape de l'évolution haïtienne, Port-au-Prince, Imprimerie La Presse.

-, 1952, «Folk-Lore et Patriotisme. Conférence au nom de l'Alliance française le 24 novembre

1951 ", Revue de la Société Haïtienne d'Histoire, de Géographie et de Géologie, vol. 28, no 84 , p. 1-16.

-, 1962, De la préhistoire d'Afrique à l'histoire d'Haïti, Port-au-Prince, Imprimerie de l'État.

-, 1971, "Discours prononcé par le Dr Jean Price-Mars », Conjonction, no 115.

PRICHARD Hesketh, 1900, Where Black Rules White; A Journey Across and about Hayti,

Westminster, Archibald Constable and Co.

Reinach Salomon, 1909, Orpheus. Histoire générale des religions, Paris, Alcide Picard.

Robert Paul (Mgr), 1955, Positions et propositions, Gonaïves.

Roumain Jacques, 1931, La montagne ensorcelée, Port-au-Prince, Imprimerie E. Chassagne. -, 1944, Les Gouverneurs de la rosée, Port-au-Prince, Imprimerie de l'État.

SAINT-John Spenser, 1886, Haïti ou la République Noire, Paris, E. Plon, Nourrit et Cie, Imprimeurs-Éditeurs.

SoufFrant Claude, 1988 (15-21 juin), "Jean Price-Mars et sa révolution culturelle ", Haïti en marche, p. 12.

STEIN Ludwig, 1897, Origine psychique et caractère sociologique de la religion. Extrait de la Revue Internationale de Sociologie, Paris, V. Giard et E. Brière Libraires-Éditeurs. Sylvain Bénito, 1894 (16 décembre), "Le Petit Parisien et la République d’Haïti ", La Fraternité, $\mathrm{n}^{\circ}$ 6, p. 4.

Texier Charles, 1891, Au pays des généraux : Haïti, Paris, Calmann-Lévy.

Thomas Dorilas (Général), 1912 (13 avril), "Boîte aux Lettres. Hommage au Président ", Le Nouvelliste, $\mathrm{n}^{\circ} 4097, \mathrm{p} .2$. 
Trouillot Duverneau, 1885, Esquisse ethnographique. «Le Vaudoun », Port-au-Prince, Imp. Ritt. Ethéart.

Trouillot Michel-Rolph, 1993, " Jeux de mots, jeux de classe. Les mouvances de l'indigénisme ", Conjonction, no 197, p. 29-41.

Trousset Jules (dir.), s.d., Le Nonvean Dictionnaire Encyclopédique Universel Illustré, vol. 5, Paris, Librairie Illustrée.

TYLOR Edward B., 1873, Primitive Culture: Researches into the Development of Mythology, Philosophy, Religion, Language, Art, and Custom. 2 Vol., Londres, John Murray.

VIncEnt Sténio, 1910, La République d'Haïti telle qu'elle est. Aperçus : Historique, Géographique, Ethnographique, Politique, Administratif, Agricole, Pastoral, Économique, Minier, Intellectuel, Législatif, Financier, Médicinal, Patriotique, Bruxelles, Société Anonyme Belge d'Imprimerie.

\section{Documentaire vidéo}

Talents Canadiens, numéro 3. Chansons Créoles avec Andrée Lescot. Film documentaire réalisé par Roger Blais de l’Office National du Film, 1952.

\section{La démonstration durkheimienne de Jean Price-Mars : faire du vodou haïtien une religion}

Jusqu'aux années 1920, le vodou haïtien était généralement considéré comme un tissu de superstitions, de sorcellerie et de pratiques rituelles cannibales. Cette situation a généré des campagnes antisuperstitieuses, appuyées par les élites gouvernementales et intellectuelles, pour éradiquer ce signe de primitivité faisant obstacle à la civilisation. En 1928, quand Jean Price-Mars publie Ainsi parla l'Oncle, il signe non seulement la naissance de l'indigénisme et de la négritude, mais aussi la réhabilitation du vodou en Haïti. Dans ce cadre, l'application du concept de "religion », tel qu'envisagé dans Les formes élémentaires de la vie religieuse d'Émile Durkheim (1912), a suscité une progression intellectuelle chez Jean Price-Mars et un tournant dans l'histoire culturelle en Haïti.

Mots-clés : Jean Price-Mars, Haïti, vodou, Émile Durkheim, religion, superstition.

\section{The durkheimian demonstration of Jean Price-Mars: To turn Haitian voodoo into a religion}

Until the 1920s, Haitian voodoo was generally regarded as a string of superstitions, witchcraft and ritual cannibalism. This situation generated anti-superstition campaigns, supported by the governmental and intellectual elites, to eradicate the signs of primitiveness obstructing civilization. In 1928, the publication of Jean PriceMars's So Spoke the Uncle led not only to the birth of Indigenism and Negritude, but also to the rehabilitation of voodoo in Haiti. In this context, applying the concept of "religion", as envisaged in Les formes élémentaires de la vie religieuse by Émile Durkheim (1912), brought about an intellectual breakthrough for Price-Mars and a turning point in the cultural history in Haiti.

Key words: Jean Price-Mars, Haiti, voodoo, Émile Durkheim, religion, superstition. 


\section{La prueba durkheimiana de Jean Price-Mars: hacer del vudú haitiano una religión}

Hasta la década de 1920, el vudú haitiano fue considerado como un tejido de supersticiones, brujería y canibalismo ritual. Esta situación generó campañas de lucha contra la superstición, apoyadas por las élites gubernamentales e intelectuales, para erradicar este signo de primitivismo que obstaculizaba la civilización. En 1928, cuando Jean Price-Mars publica Así lo dijo el tío, él provocó no sólo el nacimiento del indigenismo y la negritud, sino también la rehabilitación del vudú en Haití. En este contexto, la aplicación del concepto de "religión", tal como se aborda en Las Formas elementales de la vida religiosa de Emile Durkheim (1912), suscitó tanto la progresión intelectual de Jean Price-Mars como un viraje en la historia cultural en Haití.

Palabras clave: Jean Price-Mars, Haití, vudú, Émile Durkheim, religión, superstición. 